\title{
International Facility Comparison Campaign at L/C Band Frequencies
}

\author{
M.A.Saporetti, L.J. Foged
}

A. A. Alexandridis
F. las Heras

C. López

\author{
Y. Kurdi, M. Sierra Castañer
}

\begin{abstract}
Comparison activities in which a number of measurement facilities compare their measurements of the same antenna in a standard configuration have become important for documentation and validation of laboratory expertise and competence. It is also mandatory to have regular participation in such activities to obtain and maintain accreditations like ISO 17025. The main goal of the facility comparison activities is to provide a formal opportunity for the participants to validate and document their achieved measurement accuracy and procedures by comparison with other facilities.

Since 2004, comparison campaigns with different scopes have been conducted on antenna measurements within various European activities. Results of these activities have led to improvement in antenna measurement procedures and protocols in facilities and contributions to standards.

In this paper we report on a recent EurAAP facility comparison campaign involving a medium gain ridge horn, MVI-SH800. The antenna is equipped with an absorber plate to reduce the sensibility to the measurement set-up in order to evaluate the improvement with respect to the previous ACE intercomparison activity, involving the same antenna lacking of absorber plate. The campaign covers measurement in the $L$ and $C$ band frequencies in different facilities in Europe and USA.
\end{abstract}

\section{INTRODUCTION}

Comparison activities in which a number of measurement facilities compare their measurements of the same antenna in a standard configuration have become important for documentation and validation of laboratory expertise and competence. It is also mandatory to have regular participation in such activities to obtain and maintain accreditations like ISO 17025 [1]. The main goal of the facility comparison activities is to provide a formal opportunity for the participants to validate and document their achieved measurement accuracy and procedures by comparison with other facilities. In fact, the measurement of any antenna performance parameter is considered to be incomplete without knowledge of the measurement accuracy [2], [3].

Since 2004, comparison campaigns with different scopes have been conducted on antenna measurements within various European activities: EurAAP (European Association on Antennas and Propagation) [4] supported by the European Cooperation in Science and Technology (COST) in the programs ASSIST IC0603 [5] and VISTA IC1102 [6] and the 6th EU framework network "Antenna Centre of Excellence" (ACE) [7]. Results of these activities have led to improvement in antenna measurement procedures and protocols in facilities and contributions to standards. Due to the direct benefits to the participants, the activities have been very successful and partial results have been published in IEEE referenced papers during the years ([8]-[24]). The large amount of measured data available have fostered fruitful discussion and research on the improvement of standard procedures, protocols and tools for performance verification like the facility comparison campaigns. As a further benefit, the campaigns have initiated a dialogue among different laboratories throughout Europe and USA and is spreading into Asia.

In this paper we report a recent EurAAP facility comparison campaign involving a medium gain ridge horn, MVI-SH800. The campaign covers measurement in the $\mathrm{L}$ and $\mathrm{C}$ band frequencies in different facilities in Europe and USA. The antenna is equipped with an absorber plate to enhance the correlation in different facilities by reducing the sensibility to the measurement set-up, in order to evaluate the improvement with respect to the previous $\mathrm{ACE}$ intercomparison activity ([8],[13]), involving the same antenna lacking of absorber plate. During that campaign, in fact, the measurement set-up was without absorbers and each facility provided the necessary absorbers to cover the antenna positioner. The results of 7 facilities will be shown in terms of gain/directivity patterns, 
equivalent noise level and the declared uncertainty will be checked against the whole set of measurements.

\section{MEASUREMENTS INTERCOMPARISON}

The intercomparison campaigns have fostered a fruitful discussion on the modernization and harmonization of the techniques to intercompare different measurements of the same antenna. The data elaborations strategies have recently been revised in [24] with the focus on the determination of the reference pattern, obtained from several independent measurements, and the equivalent noise level, intended as the correlation between the reference pattern and each measurement.

\section{A. Reference Pattern}

According to [24], a reference pattern can be computed using a weighted linear mean:

$$
\mu_{\text {Lin }}=\frac{\sum_{i=1}^{n} w_{i} x_{i \text { Lin }}}{\sum_{i=1}^{n} w_{i \text { Lin }}}
$$

where:

$$
\begin{aligned}
& n=\text { total number of participants (and of measurements), } \\
& i=\text { measurement of the } i t h \text { participant to the campaign, } \\
& x_{\text {iLin }}=\text { linear measurement. }
\end{aligned}
$$

The value for the weight $w_{i}$ associated to the $i^{\text {th }}$ measurement is given by:

$$
w_{i \text { Lin }}=\frac{1}{\sigma_{i \text { Lin }}^{2}}
$$

$\sigma_{\text {Lin }}$ is the linear uncertainty computed starting from $\sigma_{d B}$ that is the uncertainty, related to the measurement, declared by each facility.

\section{B. Equivalent Noise Level}

The correlation between each measurement and the reference pattern can be expressed though the equivalent "noise" level (EQN), evaluated, in $\mathrm{dB}$, on a limited $\left( \pm 45^{\circ}\right.$ or $\pm 60^{\circ}$ ) theta cone, with the following expression:

$$
E q N_{d B}=\left[R M S E\left(\frac{D i r_{c o, x p}-D_{i r} r_{r e f \_c o, x p}}{D i r_{c o, r e f \_b o r e s i g h t}}\right)\right]
$$

where:

RMSE is the Root Mean Square Error,
$D i r_{c o, c x}=$ Directivity $(\mathrm{Co}$ or $\mathrm{Cx})$ of the measured pattern,

$D_{i r_{r e f} c o, c x}=$ Directivity of the reference pattern $(\mathrm{Co}$ or $\mathrm{Cx})$, Dir co, ref,boresight $=$ Directivity of the co-polar component of the reference pattern in boresight.

During the previous ACE campaign involving the SH800 without absorber plate, the Standard Deviation has been used instead of RMSE. In case of good correlation, both give similar values of EQN. The Standard Deviation presented in [8][11] corresponds to (3) in a linear form.

When gain is available, one can compute the equivalent noise level with the following:

$$
E q N=\left[R M S E\left(\frac{\text { GainOffset }_{c o, x p}-\text { Gain }_{r e f \_c o, x p}}{\text { Gain }_{c o, r e f_{-} \text {boresight }}}\right)\right]
$$

where:

$$
\text { GainOffset }_{d B}=\text { Gain }_{d B}-\text { Offset }_{d B}
$$

and the offset is given by:

$$
\text { Offset }_{d B}=\text { Gain }_{d B, c o_{-} \text {boresight }_{1}}-\text { Gain }_{d B, c o, \text { ref_boresight }_{-}}
$$

\section{MVI SH800 WITH ABSORBERS PLATE}

\section{A. Test object}

MVI SH800 is a Dual-Ridge Horn which combines stable gain performance and low VSWR with wideband frequency operation. The horn is single linearly polarized with excellent cross-polar discrimination. The unique horn design suppresses any possible excitation of higher order modes in the aperture and maintains a well-defined smooth radiation pattern in the direction of the boresight axis throughout the operational bandwidth. In this campaign, the antenna has been modified in order to have a more stable setup. In particular, an absorber plate has been added behind the antenna to enhance the correlation in different facilities by reducing the sensibility to the measurement set-up, as shown in Figure 1.
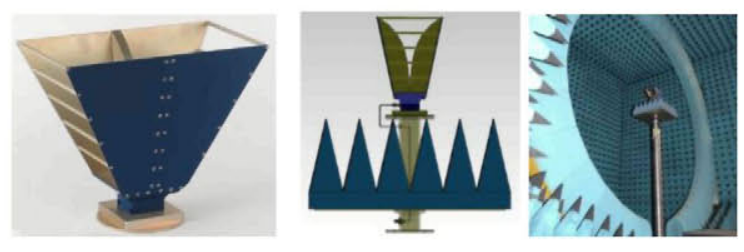

Figure 1. MVI SH800 with absorbers plate.

\section{B. Measurement Campaign}

The facilities that took part to the intercomparison and whose data are presented in this paper are:

- MVG Stargate64 in Atlanta-USA

- MVG Stargate64 in Paris, France

- Universidad de Oviedo, Spain

- Un. Politécnica de Madrid (UPM), Spain 
- IMST, Germany

- NCSR Demokritos, Institute of Informatics \& Telecommunications (NCSRD), Greece

- Inst.of High Frequency Technology, RWTH Aachen, Germany.

Figure 2. shows the location of the facilities with the indication of the type of measurement system.

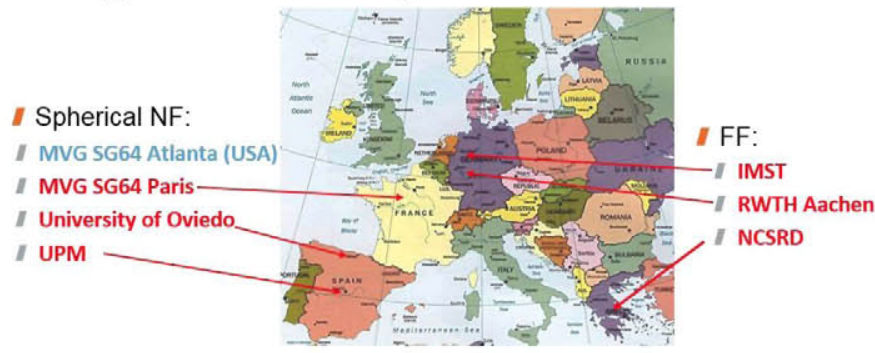

Figure 2. Involved facilities in the SH800+absorbers plate campaign.

\section{Test plan} Table I.

The test plan and type of measurements are reported in

TABLE I. TEST PLAN FOR SH800 CAMPAIGN.

\begin{tabular}{|c|l|c|}
\hline \multirow{2}{*}{$\begin{array}{c}\text { Full } \\
\text { 3D } \\
\text { Gain } \\
\begin{array}{c}\text { Meas } \\
\text { ureme } \\
\text { nt }\end{array}\end{array}$} & Freq. & $\begin{array}{c}{[0.8-1] \mathrm{GHz},[1.5-2] \mathrm{GHz},[2.2-2.7] \mathrm{GHz}} \\
\text { step } 10 \mathrm{MHz} \text { for Freq } \leqslant 1 \mathrm{GHz} \\
\text { step } 20 \mathrm{MHz} \text { for Freq }>1 \mathrm{GHz}\end{array}$ \\
\cline { 2 - 3 } & Phi & $\begin{array}{l}\text { P } 4.5 \mathrm{GHz} \\
{[4.9-6] \mathrm{GHz}, \text { step } 100 \mathrm{MHz}}\end{array}$ \\
\cline { 2 - 3 } & Theta & From $0^{\circ}$ to $135^{\circ}\left(45^{\circ}\right.$ step $)$ \\
\hline
\end{tabular}

IV. RESULTS

\section{A. Gain}

The results that will be shown hereafter are referred to the gain patterns measured by: MVG SG64 Paris, MVG SG64 Atlanta, UPM, IMST, NCSRD and Oviedo. The weighted gain reference pattern has been computed according to the $2 \sigma$ uncertainties reported in Table II. excluding University of Oviedo, whose uncertainty is under revision.

TABLE II. FACILITIES AND UNCERTAINTIES FOR THE REFERENCE GAIN PATTERN COMPUTATION

\begin{tabular}{|l|c|c|c|c|}
\hline \multirow{2}{*}{\multicolumn{1}{|c|}{ Facility }} & \multicolumn{5}{c|}{ Gain Uncertainty 2 } \\
\cline { 2 - 5 } & $\mathbf{1 8 0 0}$ & $\mathbf{2 5 0 0}$ & $\mathbf{4 0 0 0}$ & $\mathbf{5 0 0 0}$ \\
\hline MVG SG64 Paris & 0.6 & 0.6 & 0.6 & 0.6 \\
\hline MVG SG64 Atlanta & 0.6 & 0.6 & 0.6 & 0.6 \\
\hline UPM & 0.16 & 0.16 & 0.16 & 0.16 \\
\hline IMST & 0.2 & 0.2 & 0.2 & 0.2 \\
\hline NCSRD & 1.05 & 1.05 & 1.05 & 1.05 \\
\hline Oviedo & $*$ & $*$ & $*$ & $*$ \\
\hline
\end{tabular}

\section{1) Gain Radiation patterns}

Measured co-polar and cross-polar gain patterns at $\mathrm{phi}=0^{\circ}$ and $90^{\circ}$ at $1.8 \mathrm{GHz}$ are compared with the weighted reference pattern, computed with (1), in Figure 3 and Figure 4.

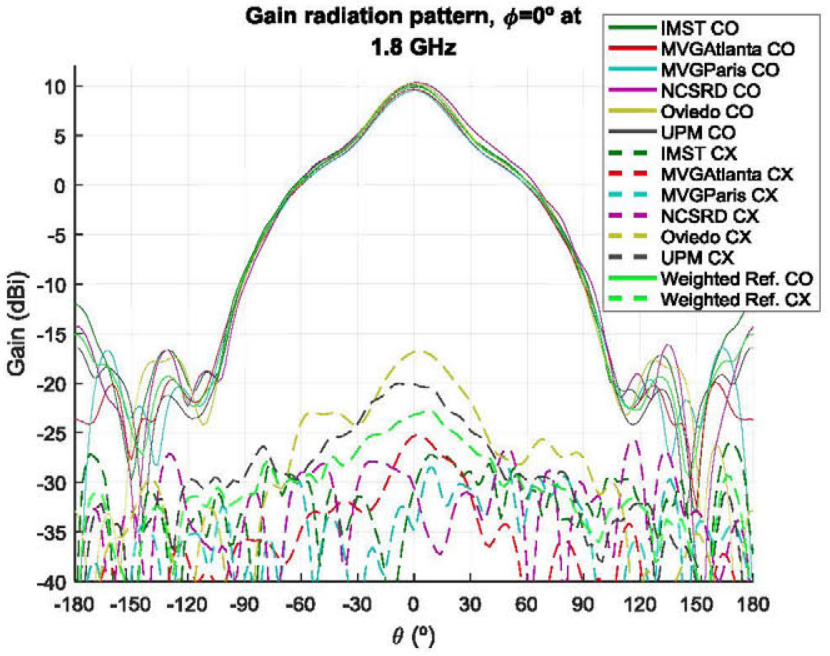

Figure 3. Gain radiation pattern at phi=0 $@ 1.8 \mathrm{GHz}$.

Weighted reference, MVG Paris, MVG Atlanta, UPM, IMST, NCSRD, Oviedo.

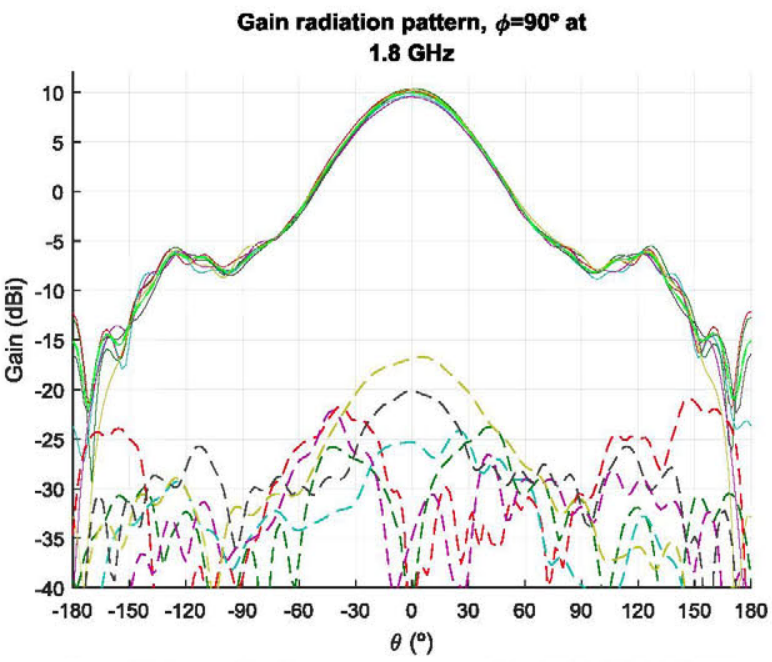

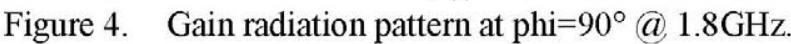

Weighted reference, MVG Paris, MVG Atlanta, UPM, IMST, NCSRD, Oviedo (see legend of Figure 3).

\section{2) Equivalent Noise Level}

The EQN computed with offset gain patterns (4) in a $\pm 45^{\circ}$ theta cone is reported in Figure 5 and Figure $6 @ 1.8,2.5,4$ and $5 \mathrm{GHz}$, computed at phi $=0^{\circ}$ and phi $=90^{\circ}$ planes for the co-polar component. The values of the peak IEEE gain are reported in Table III. together with the difference (in red) with respect to the REF. The EQN as a function of theta at $1.8 \mathrm{GHz}$ is shown for the co-polar components at $\mathrm{phi}=0^{\circ}$ and $\mathrm{phi}=90^{\circ}$ for all facilities in Figure 7 and Figure 8. 


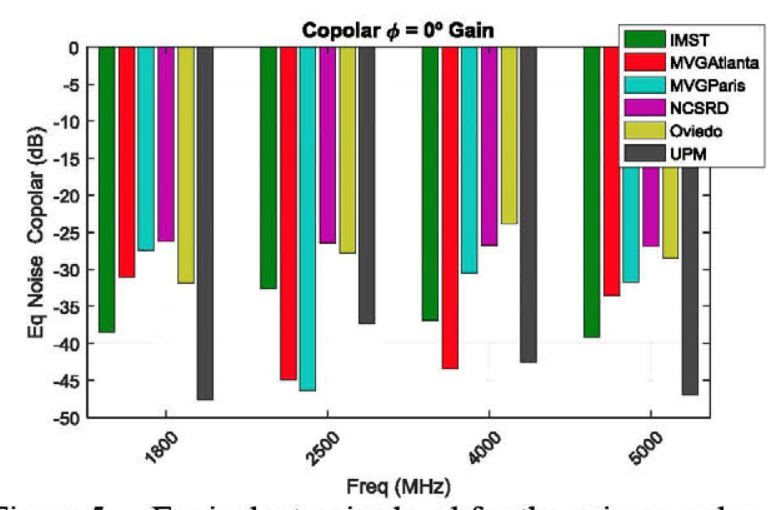

Figure 5. Equivalent noise level for the gain co-polar component at $\mathrm{phi}=0^{\circ}$.

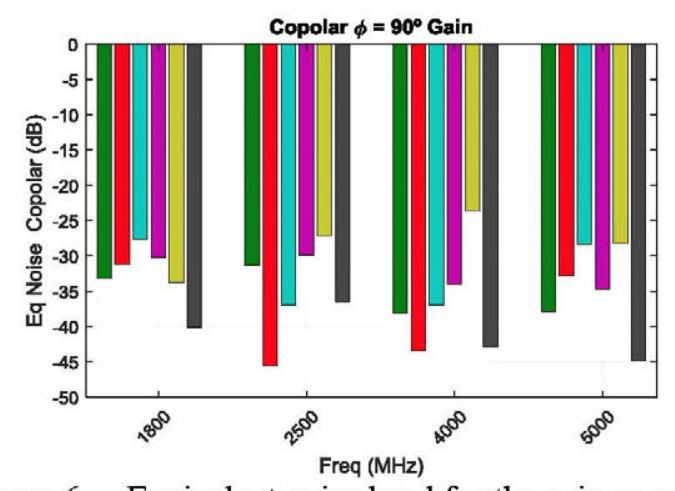

Figure 6. Equivalent noise level for the gain co-polar component at phi $=90^{\circ}$ (see legend of Figure 5).

TABLE III. PEAK IEEE GAIN

\begin{tabular}{|c|c|c|c|c|c|c|c|}
\hline \multirow{2}{*}{$\begin{array}{c}\text { Freq } \\
{[\text { GHz] }}\end{array}$} & \multicolumn{7}{|c|}{ Peak Gain } \\
\hline & $R E F$ & $\begin{array}{c}M V G \\
P\end{array}$ & $\begin{array}{c}M V G \\
A\end{array}$ & $U P M$ & IMST & $\begin{array}{c}N C S R \\
D\end{array}$ & $O V I$ \\
\hline 1.8 & 9.96 & $\begin{array}{c}9.52 \\
-0.44\end{array}$ & $\begin{array}{r}9.67 \\
-0.29\end{array}$ & $\begin{array}{c}9.91 \\
-0.05\end{array}$ & $\begin{array}{c}10.12 \\
0.16\end{array}$ & $\begin{array}{c}10.33 \\
0.37\end{array}$ & $\begin{array}{c}10.27 \\
(0.31)^{*}\end{array}$ \\
\hline 2.5 & 10.38 & $\begin{array}{l}10.35 \\
-0.03\end{array}$ & $\begin{array}{l}10.42 \\
0.06\end{array}$ & $\begin{array}{l}10.22 \\
-0.16\end{array}$ & $\begin{array}{c}10.63 \\
0.25\end{array}$ & $\begin{array}{c}10.74 \\
0.36\end{array}$ & $\begin{array}{c}10.89 \\
(0.51)^{*}\end{array}$ \\
\hline 4 & 10.78 & $\begin{array}{r}10.60 \\
-0.18\end{array}$ & $\begin{array}{l}10.81 \\
0.03\end{array}$ & $\begin{array}{l}10.73 \\
-0.05\end{array}$ & $\begin{array}{c}10.87 \\
0.09\end{array}$ & $\begin{array}{c}10.82 \\
0.04\end{array}$ & $\begin{array}{r}11.56 \\
(0.78)^{*}\end{array}$ \\
\hline 5 & 11.6 & $\begin{array}{l}11.26 \\
-0.34\end{array}$ & $\begin{array}{l}11.47 \\
-0.13\end{array}$ & $\begin{array}{l}11.56 \\
-0.04\end{array}$ & $\begin{array}{c}11.72 \\
0.12\end{array}$ & $\begin{array}{c}11.69 \\
0.09\end{array}$ & $\begin{array}{c}12.09 \\
(0.49)^{*}\end{array}$ \\
\hline
\end{tabular}

*the REF has been computed excluding University of Oviedo

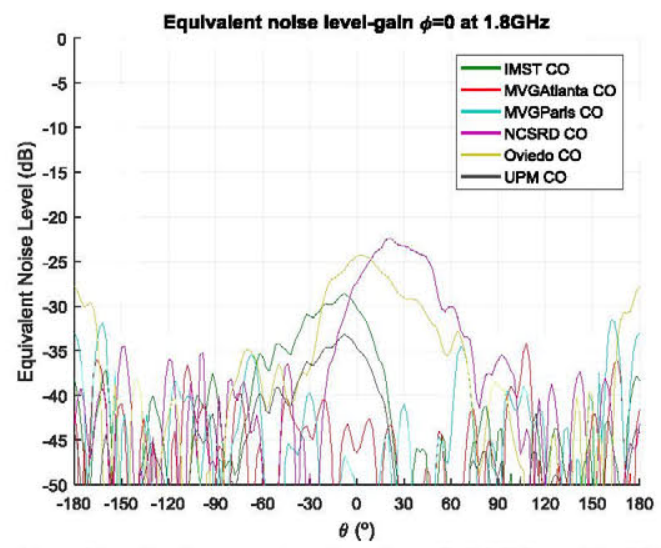

Figure 7. Equivalent noise level at $1.8 \mathrm{GHz}$, phi $=0^{\circ}$ w.r.t. theta for the gain co-polar component.

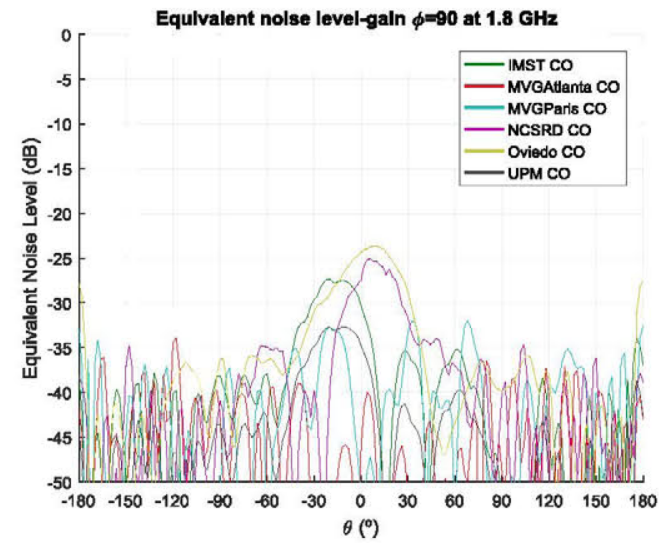

Figure 8. Equivalent noise level at $1.8 \mathrm{GHz}$, phi $=90^{\circ}$ w.r.t. theta for the gain co-polar component.

\section{B. Directivity}

The results that will be shown hereafter are referred to the directivity patterns measured by: MVG SG64 Paris, MVG SG64 Atlanta, UPM, NCSRD, Oviedo, RWTH Aachen.

The weighted directivity reference pattern has been computed according to the $2 \sigma$ uncertainties reported in Table IV. excluding Oviedo, whose uncertainty is under revision.

TABLE IV. FACILITIES AND UNCERTAINTIES FOR THE REFERENCE DIRECTIVITY PATTERN COMPUTATION

\begin{tabular}{|c|c|c|c|c|}
\hline \multirow{2}{*}{ Facility } & \multicolumn{4}{|c|}{$\begin{array}{c}\text { Directivity Uncertainty } 2 \sigma(a) \\
\text { freq }[\mathrm{MHz}]\end{array}$} \\
\hline & 1800 & 2500 & 4000 & 5000 \\
\hline MVG SG64 Paris & 0.3 & 0.3 & 0.3 & 0.3 \\
\hline MVG SG64 Atlanta & 0.3 & 0.3 & 0.3 & 0.3 \\
\hline UPM & 0.1 & 0.1 & 0.1 & 0.1 \\
\hline RWTH Aachen & - & 0.20 & 0.20 & 0.16 \\
\hline NCSRD & 1.06 & 1.06 & 1.06 & 1.06 \\
\hline Oviedo & $*$ & * & $*$ & $*$ \\
\hline
\end{tabular}

\section{1) Directivity Radiation patterns}

Measured co-polar and cross-polar directivity patterns at phi $=0^{\circ}$ and $90^{\circ}$ at $5 \mathrm{GHz}$ are compared with the weighted reference pattern, computed with (1), in Figure 9 and Figure 10.

\section{Equivalent Noise Level}

The EQN computed in a $\pm 45^{\circ}$ theta cone, using (3) is reported in Figure 11 and Figure 12 at 1.8, 2.5, 4 and $5 \mathrm{GHz}$, computed at $\mathrm{phi}=0^{\circ}$ and $\mathrm{phi}=90^{\circ}$ planes for the co-polar component. The values of the peak directivity are reported in Table V. together with the difference (in red) with respect to the REF. The EQN as a function of theta at $5 \mathrm{GHz}$ is shown for the co-polar components at $\mathrm{phi}=0^{\circ}$ and $\mathrm{phi}=90^{\circ}$ for all facilities in Figure 13 and Figure 14. 


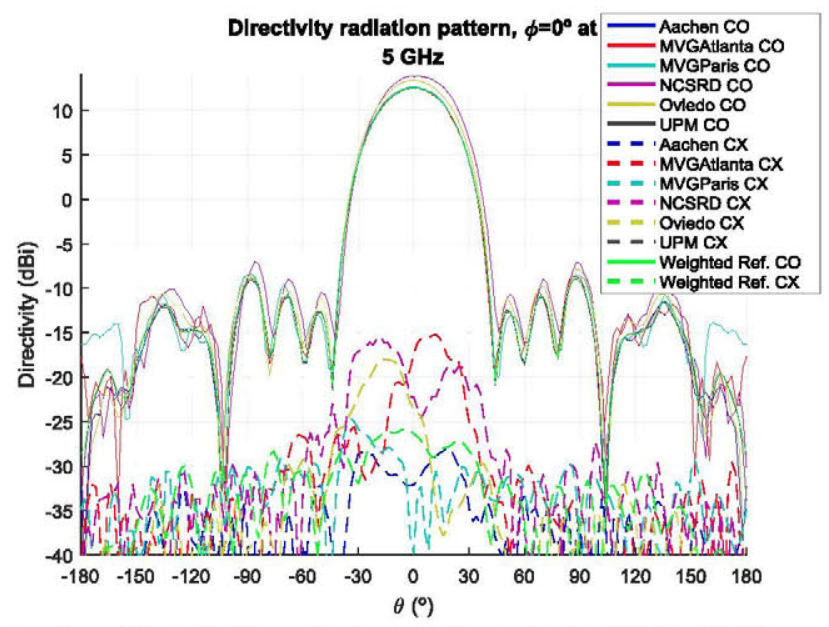

Figure 9. Directivity radiation pattern at $\mathrm{phi}=0^{\circ} @ 5 \mathrm{GHz}$.

Weighted reference, MVG Paris, MVG Atlanta, UPM, NCSRD, Oviedo, RWTH Aachen.

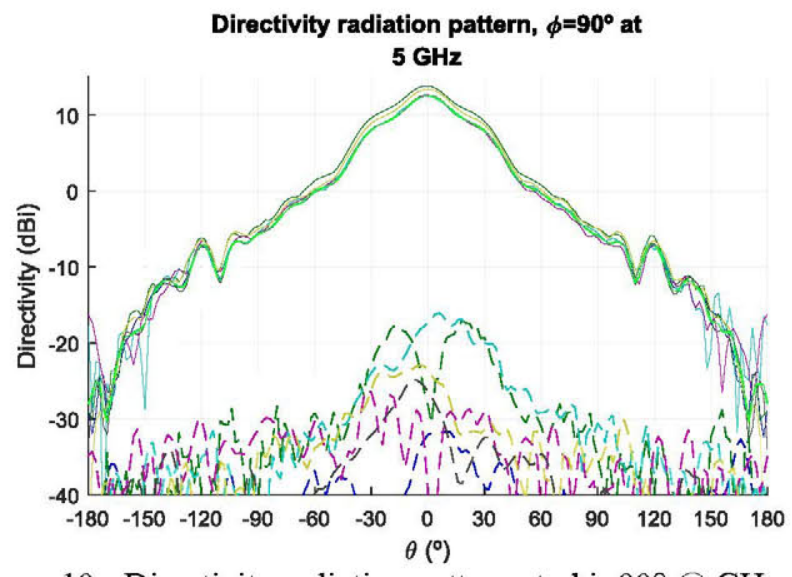

Figure 10. Directivity radiation pattern at phi $=90^{\circ} @ \mathrm{GHz}$.

Weighted reference, MVG Paris, MVG Atlanta, UPM,

NCSRD, Oviedo, RWTH Aachen (see legend of Figure 9).

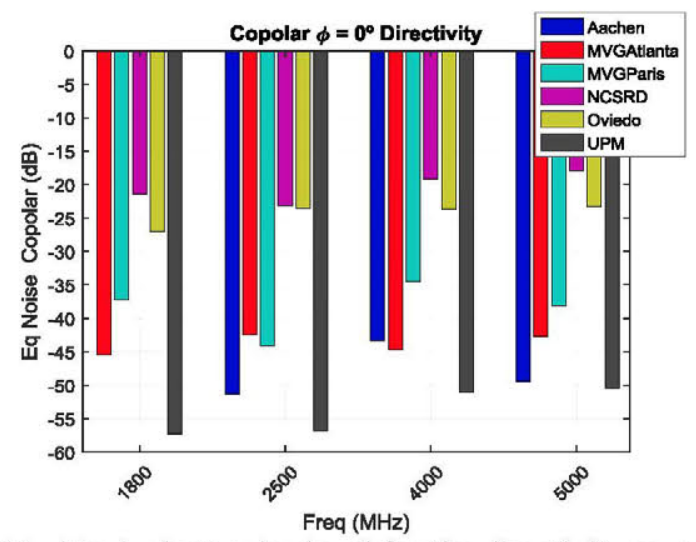

Figure 11. Equivalent noise level for the directivity co-polar component at $\mathrm{phi}=0^{\circ}$.

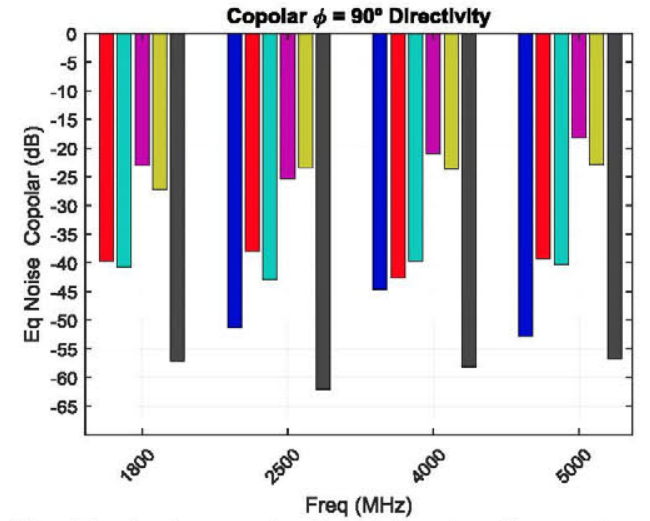

Figure 12. Equivalent noise level for the directivity co-polar component at phi $=90^{\circ}$ (sse legend of Figure 11).

TABLE V. PEAK DIRECTIVITY

\begin{tabular}{|c|c|c|c|c|c|c|c|}
\hline \multirow{2}{*}{$\begin{array}{c}\text { Freq } \\
\text { [GHz] }\end{array}$} & \multicolumn{7}{|c|}{ Peak Directivity } \\
\hline & $R E F$ & $\begin{array}{c}M V G \\
P\end{array}$ & $\begin{array}{c}M V G \\
A\end{array}$ & $U P M$ & $\begin{array}{c}\text { Aache } \\
n\end{array}$ & $\begin{array}{c}N C S R \\
D\end{array}$ & $O V I$ \\
\hline 1.8 & 10.5 & $\begin{array}{r}10.44 \\
-0.06\end{array}$ & $\begin{array}{c}10.54 \\
0.04\end{array}$ & $\begin{array}{c}10.5 \\
0\end{array}$ & - & $\begin{array}{c}11.32 \\
0.82\end{array}$ & $\begin{array}{c}11.05 \\
(0.55)^{*}\end{array}$ \\
\hline 2.5 & 11.24 & $\begin{array}{l}11.22 \\
-0.02\end{array}$ & $\begin{array}{c}11.33 \\
0.09\end{array}$ & $\begin{array}{c}11.23 \\
-0.01\end{array}$ & $\begin{array}{l}11.29 \\
0.05\end{array}$ & $\begin{array}{r}11.90 \\
-0.66\end{array}$ & $\begin{array}{l}12.04 \\
(0.8)^{*}\end{array}$ \\
\hline 4 & 11.62 & $\begin{array}{c}11.65 \\
0.03\end{array}$ & $\begin{array}{l}11.61 \\
-0.01\end{array}$ & $\begin{array}{r}11.6 \\
-0.02\end{array}$ & $\begin{array}{c}11.68 \\
0.06\end{array}$ & $\begin{array}{c}12.46 \\
0.84\end{array}$ & $\begin{array}{c}12.38 \\
(0.76)^{\circ}\end{array}$ \\
\hline 5 & 12.53 & $\begin{array}{c}12.63 \\
0.1\end{array}$ & $\begin{array}{c}12.65 \\
0.12 \\
\end{array}$ & $\begin{array}{r}12.49 \\
-0.04 \\
\end{array}$ & $\begin{array}{c}12.56 \\
0.03\end{array}$ & $\begin{array}{c}13.80 \\
1.27\end{array}$ & $\begin{array}{c}13.38 \\
(0.85)^{*}\end{array}$ \\
\hline
\end{tabular}

\section{CONCLUSIONS}

The results of a facility comparison campaign involving a medium gain ridge horn, MVI-SH800, working at L and C band frequencies and equipped with an absorber plate to reduce the sensibility to the measurement set-up, have been presented. The antenna has been measured in 7 different facilities in Europe and USA, in the frame of the intercomparison activities based on high accuracy reference antennas supported by EurAAP, which have fostered fruitful discussion on the improvement of standard procedures for performance verification like facility comparison campaigns.

The measurements from the 7 different facilities are generally in very good agreement when compared to each other. The visible pattern agreement is confirmed by the equivalent noise level (pattern correlation) of less than $\sim-30$ $\mathrm{dB}$. Very good agreement has been achieved also for performance parameters such as peak directivity and peak gain. Such results confirm the expected improvement with respect to the previous SH800 campaign (without absorber plate) where standard deviation errors were $\sim 0.05$ which corresponds to an $\mathrm{EQN}$ of $-\sim 26 \mathrm{~dB}$. 


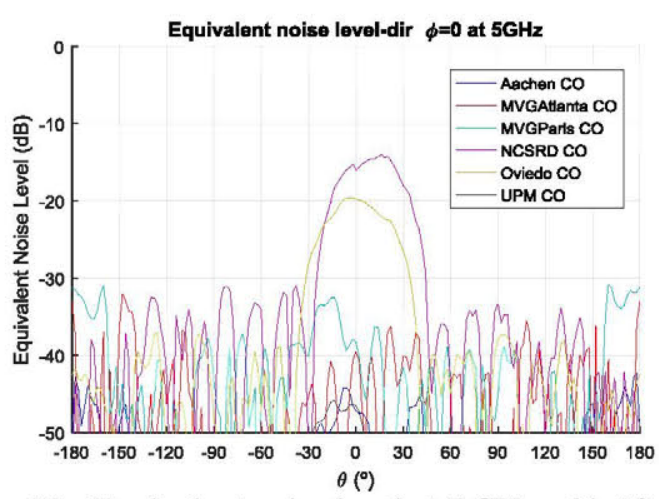

Figure 13. Equivalent noise level at $5 \mathrm{GHz}$, phi $=0^{\circ}$ w.r.t. theta for the directivity co-polar component.

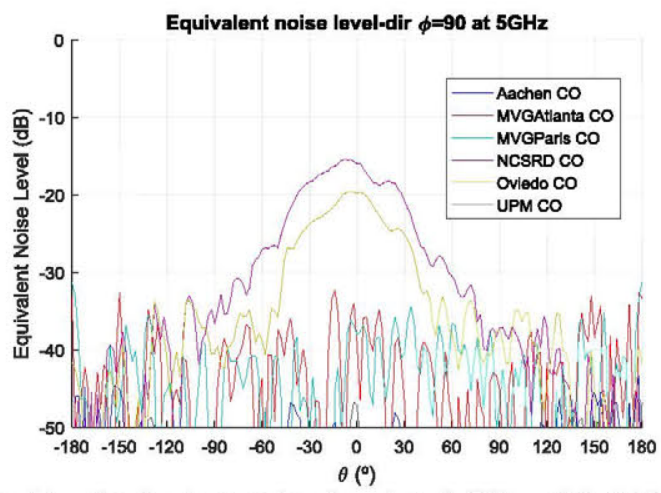

Figure 14. Equivalent noise level at $5 \mathrm{GHz}$, phi $=90^{\circ}$ w.r.t. theta for the directivity co-polar component.

\section{ACKNOWLEDGEMENT}

This work has been partially supported by the Spanish project ENABLING5G "Enabling Innovative Radio Technologies for 5G networks" (TEC2014-55735-C3-1-R) and by COST-VISTA and EurAAP.

\section{REFERENCES}

[1] www.isoiec17025.com

[2] ANSI/IEEE Standard 149-1979, "Test Procedures for Antennas".

[3] IEEE Standard 1720-2012, "Reccomended practices for near-field antenna measuremenst".

[4] EurAAP, "European Association on Antennas and Propagation", www.euraap.org

[5] COST ASSIST IC0603,"Antenna Systems and Sensors for Information Society Technologies", www.cost-ic0603.org

[6] COST VISTA IC1102, "Action on Versatile, Integrated and Signalaware Technologies for Antennas", www.cost-vista.eu

[7] http://www.ist-ace.org/

[8] L. J. Foged, P. Garreau, O. Breinbjerg, S. Pivnenko, M. Castañer, J. Zackrisson "Facility comparison and evaluation using dual ridge horn," AMTA 2005, Newport, USA.

[9] L. J. Foged, P. O Iversen, L. Duchesne, O. Breinbjerg, S. Pivnenko, "Comparative Measurement of standard gain horns," 28th ESA antenna workshop on Space Antenna Systems and Technologies, 31 May - 3 June 2005, Noordwijk, The Netherlands.

[10] L J. Foged, B. Bencivenga, L. Durand, O. Breinbjerg, S. Pivnenko, C. Sabatier, H. Ericsson, B Svensson, A. Alexandridis, S. Burgos, M.
Sierra-Castañer, J. Zackrisson, M. Boettcher "Error calculation techniques and their application to the Antenna Measurement Facility Comparison within the European Antenna Centre of Excellence," 2nd European Conference on Antennas and Propagation, EuCAP, $11-16$ Nov. 2007 Edinburgh, UK.

[11] L. J. Foged, B. Bencivenga, O. Breinbjerg, S. Pivnenko, M. SierraCastañer, "Measurement facility comparisons within the European antenna centre of excellence," ISAP2008, International symposium on Antennas and propagation. 27-30 October 2008. Taipei, Taiwan.

[12] S. Pivnenko, O. Breinbjerg, S. Burgos, M. Sierra-Castañer, H. Eriksson, "Dedicated Measurement Campaign for Definition of Accurate Reference Pattern of the VAST12 Antenna," 2008 AMTA Symposium.

[13] L. J. Foged, B. Bencivenga, L. Scialacqua, S. Pivnenko, O. Breinbjerg, M. Sierra-Castañer, P. C. Almena, E. Séguenot, C. Sabatier, M. Böttcher, E. Arnaud, T. Monediere, H. Garcia, D. Allenic, G. Hampton, A. Daya, "Facility comparison and evaluation using dual ridge horns," 3th European Conference on Antennas and Propagation, EuCAP, Berlin, March 2009.

[14] S. Pivnenko, J.E. Pallesen, O. Breinbjerg, M. Sierra-Castañer, P. Caballero, C. Martínez, J.L. Besada, J. Romeu, S. Blanch, J.M González-Arbesu, C. Sabatier, A. Calderone, G. Portier, H. Eriksson, J. Zackrisson. "Comparison of Antenna Measurement Facilities With the DTU-ESA $12 \mathrm{GHz}$ Validation Standard Antenna Within the EU Antenna Centre of Excellence," IEEE Transactions on Antennas and Propagation, July 2009. Vol. 57. N. 7. pp. 1863- 1878.

[15] L. J. Foged, M. Sierra Castañer, L. Scialacqua "Facility Comparison Campaigns within EurAAP," 5th European Conference on Antennas and Propagation, EuCAP, Rome, April 2011.

[16] L. Scialacqua, F. Mioc, J. Zhang, L. J. Foged, M. Sierra-Castañer, "Antenna Measurement Intercomparison Campaigns in the framework of the European Association of Antennas and Propagation," International Symposium on Antennas and Propagation, ISAP, Nanjing, China, October 2013

[17] L. J. Foged, A. Giacomini, L. Duchesne, E. Leroux, L. Sassi,, J. Mollet, "Advanced modelling and measurements of wideband horn antennas," ANTEM Saint-Malo, June 2005.

[18] M. Saporetti, L. J. Foged, L. Scialacqua, F. Saccardi, M. Mercier, J. Lui, F. Z. Xue, L. T. Chen, B.L.Bu, "Antenna Measurement System Verification by Correlation with Facility-Comparison Campaign in the Framework of the European Association of Antennas and Propagation", International Wireless Symposium, March 2014.

[19] L.J. Foged, M.A. Saporetti, M. Sierra-Castañer, E. Jørgensen, T. Voigt, F. Calvano, D. Tallini, "Measurement and Simulation of Reflector Antenna",EuCAP2015, Lisbon, April 2015.

[20] M. Sierra Castañer, L.J. Foged, M.A.Saporetti, E. Jørgensen, T. Voigt, D. Tallini , M.Orefice, G. Giordanengo, G. Dassano, M. Böttcher, A. Wien, J. M. Serna , D. Pérez de Diego, F. Calvano, "Comparison of Reflector Antenna, Measurements and Simulations", AMTA 37th Annual Meeting \& Symposium, Long Beach, California | Oct 11 - 16, 2015.

[21] M.A.Saporetti, L.J. Foged, M. Sierra Castañer, E. Jørgensen, T. Voigt, A. Michel, D. Tallini, B. Kolundzija, M. Böttcher, A. Wien, M.Orefice, G. Giordanengo, G. Dassano, J. M. Serna, F. Catedra, A. Somolinos, J. Moreno, I. Gonzales, "Measurements and Simulations Correlation of High Reliability Reflector Antenna", EuCAP2016, Davos Switzerland, April 2016.

[22] M.A.Saporetti, L.J. Foged, A. Scannavini, S. Weitz, J. Kunisch, "Measurements and Simulations of MIMO 2x2 Reference Antennas", EuCAP2016, Davos Switzerland, April 2016.

[23] G. Vanderbosch, F. Mioc, M.A.Saporetti, L.J. Foged, "Bridging the Simulations-Measurements Gap" APM, December 2016.

[24] M.A.Saporetti, L.J. Foged, M. Sierra Castañer, S. Pivnenko, R. Cornelius, D. Herberling, "Description and Preliminary Results of Antenna Measurement Facility Comparisons in EurAAP", APM, May 2017. 